\title{
STATISTICAL PROPERTIES AND INFORMATION CONTENT OF CALCIUM OSCILLATIONS
}

\author{
ALEXANDER SKUPIN \\ alexander. skupin@hmi.de \\ MARTIN FALCKE \\ falcke@hmi.de \\ Hahn-Meitner-Institut, Department of Theoretical Physics, Glienicker Str. 100, \\ 14109 Berlin, Germany
}

\begin{abstract}
Calcium is the most important second messenger in living cells serving as a critical link between a large variety of extracellular stimuli and the intracellular target. Often, the $\mathrm{Ca}^{2+}$ signal is carried by $\left[\mathrm{Ca}^{2+}\right]$ oscillations. Our recent studies have demonstrated that in contrast to traditional ideas $\mathrm{Ca}^{2+}$ oscillations do not occur by simple synchronization of channel clusters opening and closing in an oscillatory fashion but originate from microscopic fluctuation caused by the stochastic binding of the ligands $\mathrm{Ca}^{2+}$ and $\mathrm{IP}_{3}$ to the receptor's binding sites. They are orchestrated spatially on the cell level by wave nucleation. In this paper we analyze the stochastic data and show how internal properties can be determined from global observations. Further, we analyze the information content of spontaneous and stimulated oscillations.
\end{abstract}

Keywords: cell signaling; calcium oscillations; noise; information estimation.

\section{Introduction}

Calcium is a ubiquitous second messenger which regulates multiple cellular functions like gene expression, secretion, muscle contraction or synaptic plasticity. The $\mathrm{Ca}^{2+}$ signal employed by such a variety of processes is a transient increase of the intracellular concentration $[2,4,7,11,15]$. This $\left[\mathrm{Ca}^{2+}\right]$ increase is due to entry through the cell membrane and to $\mathrm{Ca}^{2+}$ release from internal storage compartments especially the endoplasmic reticulum (ER) and the sarcoplasmic reticulum. Release from these stores is a nonlinear process. It leads in many cells to the formation of spatiotemporal signals in form of waves of high $\mathrm{Ca}^{2+}$ concentration travelling across the cell and global oscillations [7]. The information transmitted by these signals arrives as a stimulus at the plasma membrane and is translated into intracellular $\mathrm{Ca}^{2+}$ oscillations.

$\mathrm{Ca}^{2+}$ release from storage compartments is controlled by channels. A channel type present in the ER membrane of many cells is the inositol 1,4,5-trisphosphate $\left(\mathrm{IP}_{3}\right)$ receptor channel $\left(\mathrm{IP}_{3} \mathrm{R}\right)$. The open probability of the $\mathrm{IP}_{3} \mathrm{R}$ depends on the $\mathrm{IP}_{3}$ concentration and the calcium concentration in the cytosol (see $[7,11,14]$ for reviews). It increases with increasing $\mathrm{Ca}^{2+}$ concentration, i.e. $\mathrm{Ca}^{2+}$ release is self-amplifying. That is called Calcium Induced Calcium Release (CICR). $\mathrm{Ca}^{2+}$ released by one channel diffuses in the cytosol and increases the open probability of neighboring 
channels (see Fig. 1A). This coupling of channels by $\mathrm{Ca}^{2+}$ diffusion causes the spatial spread of release. Very high $\mathrm{Ca}^{2+}$ concentrations inhibit the channel and terminate release. Activation and inhibition together create a bell-shaped dependence of the open probability on cytosolic $\left[\mathrm{Ca}^{2+}\right]$. There is some structure in the spatial arrangement of channels: They are grouped into clusters on the ER membrane containing 1-40 channels. Clusters are randomly scattered across the ER membrane. Due to their importance and their frequent appearance, $\mathrm{Ca}^{2+}$ oscillations were the representative of cellular oscillators most prominent in mathematical physiology and modeling for the last twenty years or so. Most traditional models assume cells to behave like stirred chemical reactors, i.e. neglect concentration gradients. They describe $\mathrm{Ca}^{2+}$ dynamics by deterministic ordinary differential equations [12]. Our recent studies have shown, that intracellular $\mathrm{Ca}^{2+}$ oscillations using $\mathrm{IP}_{3}$ receptor channels are sequences of random spikes. Spikes are initiated by the local stochastic behavior of ion channels and transformed into a global $\mathrm{Ca}^{2+}$ transient by wave nucleation, i.e. by a spatial phenomenon.

Wave nucleation can be explained by the hierarchical picture of intracellular $\mathrm{Ca}^{2+}$ dynamics. It arose upon observation of random release events from single channel clusters - called puffs $[3,5,7,9,10]$. They can be considered as the elemental events of intracellular $\mathrm{Ca}^{2+}$ dynamics. According to the hierarchical concept, random opening of a single channel in a cluster causes the other channels of the same cluster to open thus generating a puff. That puff may cause neighboring clusters to open, too. If a supercritical number of puffs arises - a supercritical wave nucleus release spreads through the whole cell. The existence of such a critical nucleus was shown experimentally and theoretically $[6,9,10]$. It introduces the dependence of the triggering probability of global events on the strength of spatial coupling by $\mathrm{Ca}^{2+}$ diffusion $[6]$.

We have shown recently [13] that intracellular $\mathrm{Ca}^{2+}$ oscillations exhibit interspikeinterval (ISI) distributions with an approximately linear relation between the average ISI and the standard deviation. We have measured the time course of $\mathrm{Ca}^{2+}$ concentrations during oscillations in four different cell types. Here, we analyze the data with respect to information on the properties of the specific cell and with respect to the information content of stimulated and spontaneous spike trains using the Kullback entropy as measure for information.

\section{Methods and Results}

\subsection{Experimental Methods}

We measured $\mathrm{Ca}^{2+}$ oscillations in four different cell types: spontaneous oscillations in astrocytes, microglia, in from fat tissue developed human stem cells (PLA) and by carbamyl choline $(\mathrm{CCh})$ induced oscillations in human embryonic kidney cells (HEK). Cells were loaded with distinct fluorescence dyes and illuminated with monochromatic light to measure the global cytosolic $\mathrm{Ca}^{2+}$ concentration. The resulting fluorescence light was recorded after filtering with an appropriated bandpass 

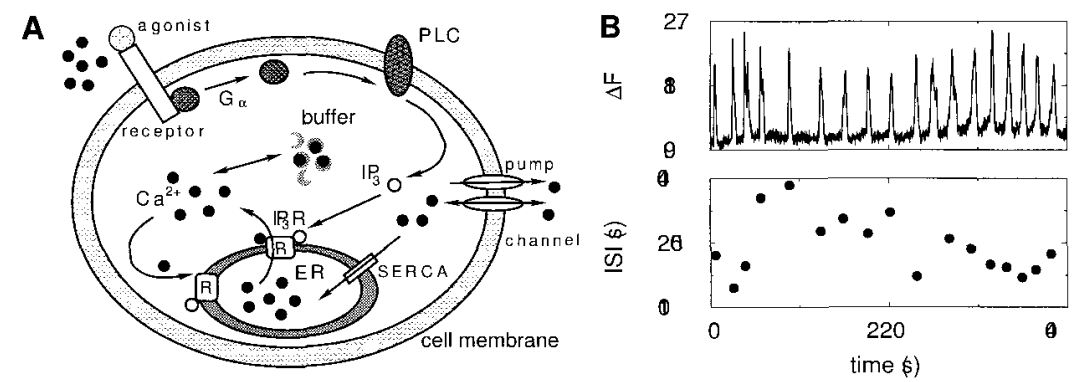

Fig. 1. A: Model of CICR. At stimulation with an agonist receptors in the cell membrane induce $\mathrm{IP}_{3}$ (circles) production by a G-protein $\left(\mathrm{G}_{\alpha}\right)$ activated PLC. If $\mathrm{IP}_{3}$ and $\mathrm{Ca}^{2+}$ (dots) are bound to the $\mathrm{IP}_{3} \mathrm{R}(\mathrm{R})$ it opens and $\mathrm{Ca}^{2+}$ diffuses from the ER into the cytosol. There it is caught by buffers and pumped back into the ER by SERCA pumps or into the extracellular space by pumps and can activate adjoining $\mathrm{IP}_{3} \mathrm{Rs}$. Some cell types express channels (gap junctions) to transmit the signal to neighboring cells. B: A typical fluorescence signal $\triangle F$ of a PLA cell is shown in the upper panel. In the lower panel for each $\mathrm{Ca}^{2+}$ spike the following ISI is shown indicating that $\mathrm{Ca}^{2+}$ oscillations have a stochastic character since the ISIs vary from about $100 \mathrm{~s}$ to $400 \mathrm{~s}$.

filter by a digital camera with sample rates between 0.2 and $0.5 \mathrm{~Hz}$.

The fluorescence signals $F$ were scaled to the initial fluorescence level $F_{0}$ leading to $\Delta \mathrm{F}=\mathrm{F} / \mathrm{F}_{0}$ shown in Fig. $1 \mathrm{~B}$ in the upper panel. We determined the interspike interval (ISI) as the time between consecutive fluorescence maxima. The lower panel of Fig. 1B shows the ISI following each spike at the time of the spike. We obtained the characteristics of the $\mathrm{Ca}^{2+}$ oscillations as the averaged ISI $\mathrm{T}_{\mathrm{av}}$ and their standard deviation $\sigma$ from these series.

In the buffer experiments, we used BAPTA or EGTA which were added to cells by loading for 5 to $10 \mathrm{~min}$. For more experimental details see [13].

The study conforms to the Declaration of Helsinki and all cell donors gave their informed written consent to use part of their fat tissue for the generation of processed lipoaspirate (PLA) cells.

\subsection{Characterizing the oscillations}

We characterize the statistical properties of these oscillations by means of the average interspike interval $\mathrm{T}_{\mathrm{av}}$ and its standard deviation $\sigma$ and their relation. The data of distinct cells and cell types are shown in Fig. 2, where $\sigma$ is plotted over $\mathrm{T}_{\mathrm{av}}$ for individual cells, each data point representing one cell. The data for astrocytes (black) and HEK cells (red) are shown in panel A and panel B contains those of microglia (black) and PLA cells (red). To quantify the different populations and for further analysis we also plotted the linear regression lines for each cell type within the corresponding colors having slopes $m_{\text {astro }}=0.93, m_{\mathrm{HEK}}=0.58, m_{\mathrm{mic}}=1.01$ and $m_{\mathrm{PLA}}=0.7$.

There are three prominent features of the data. We observe a minimal ISI as there are no oscillations with $\mathrm{T}_{\mathrm{av}}$ shorter than about $40 \mathrm{~s}$, the standard deviation $\sigma$ 

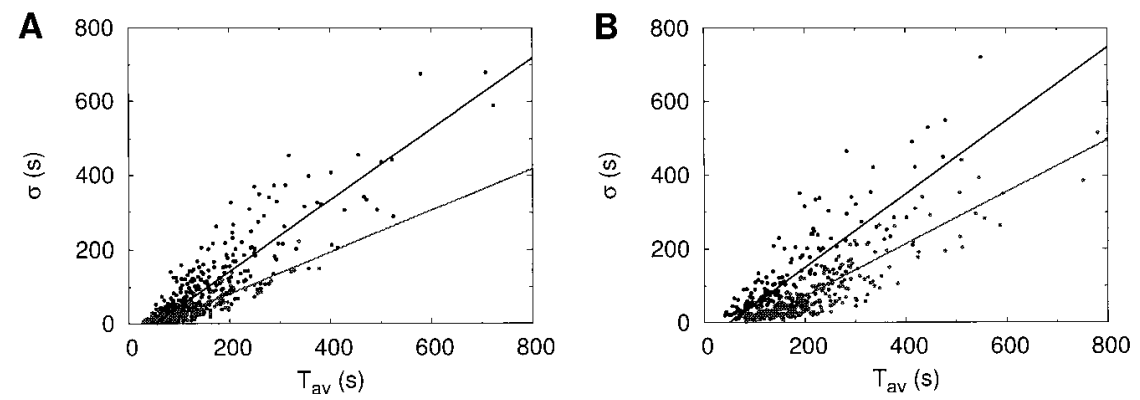

Fig. 2. $\mathrm{Ca}^{2+}$ spikes occur randomly. The standard deviation $\sigma$ of the $\mathrm{Ca}^{2+}$ oscillation are in the same range as $\mathrm{T}_{\mathrm{av}}$ shown for astrocytes (black) and stimulated HEK cells (red) in panel $\mathbf{A}$ and for microglia (black) and PLA cells (red) in panel $\mathbf{B}$ with the corresponding linear regression lines.

increases with $\mathrm{T}_{\mathrm{av}}$ and $\sigma$ reaches the same order of magnitude as $\mathrm{T}_{\mathrm{av}}$ [13]. The minimal ISI arises from the existence of a deterministic part of the ISI. It is the minimal time required for the cell to recover from a concentration spike and reflects processes like store depletion, recovery from inhibition or negative feed back of $\mathrm{Ca}^{2+}$ to $\mathrm{IP}_{3}$ production [13]. This deterministic time is specific for individual cells and thus we will refer to it as $T_{\text {cell }}$.

The fact that the standard deviations $\sigma$ are in the same range as the $\mathrm{T}_{\mathrm{av}}$ demonstrates the stochastic character of spike generation [13]. Moreover, the data structure indicates a linear dependence of $\sigma$ on $\mathrm{T}_{\mathrm{av}}$ which is in accordance with the hypothesized wave nucleation process [13]. The nucleation probability sets the stochastic part $t$ of the interspike interval. It depends on the spatial organization of cells.

We conclude from these findings that a single interspike interval is given by the cell specific recovery time and a random time $t$ as

$$
\mathrm{ISI}=\mathrm{T}_{\text {cell }}+t
$$

We denote the distribution density of $t$ by $p(t)$. The average ISI of an individual cell has a contribution $\mathrm{T}_{\text {cell }}$ and a contribution arising from $p(t)$. The latter part specifies the shape of the $\sigma-\mathrm{T}_{\mathrm{av}}$ relation

$$
\mathrm{T}_{\mathrm{av}}=\mathrm{T}_{\text {cell }}+\int_{0}^{\infty} t p(t) d t=\mathrm{T}_{\text {cell }}+\mathrm{T}_{\text {stoch }} .
$$

The spread of data points in Fig. 2 represents properties of individual cells. They differ in $\mathrm{T}_{\text {cell }}$ generating spread in the direction of $\mathrm{T}_{\mathrm{av}}$ and second in the properties of the stochastic process, shifting them in the $\sigma-\mathrm{T}_{\mathrm{av}}$ plane along curves parameterized by characteristics of the stochastic process. In the following we will try to decompose the measured $T_{a v}$ according to expression (2) into its parts $T_{\text {stoch }}$ and $T_{\text {cell }}$ to get information about the processes shaping the data.

We start with the simplifying assumption that the cell recovers completely before a spike is set off and that the probability for a nucleation event is 0 during recovery and constant after recovery. Therefore, wave nucleation is expected to obey a Poisson 
process with its characteristic waiting time distribution density

$$
p_{\text {poi }}(t)=\lambda e^{-\lambda t},
$$

where $\lambda$ denotes the wave nucleation rate. A common feature of the Poisson process is the equality of its average and standard deviation. We therefore expect that $\mathbf{T}_{\text {stoch }}$ equals $\sigma$ and is given by $\mathrm{T}_{\text {stoch }}=\sigma=1 / \lambda$, i.e. the data of cells with the same $\mathrm{T}_{\text {cell }}$ should lie on a straight line with slope one. Consequently for diverse finite $\mathrm{T}_{\text {cell }}$ the data structure should be explainable by an array of lines with slope one. For a finite number of cells and with the reasonable assumption of an upper limit for $\mathrm{T}_{\text {cell }}$ the resulting population slope might be an appropriate approximation for that relation. Indeed, we can identify in Fig. 2 such dependence for the astrocytes (panel A black) and for the microglia (panel B black) by both, inspection by eye and by consideration of the population slopes that are around one.

Hence, we can determine the cell specific time $\mathrm{T}_{\text {cell }}$ by eq. (2) by setting $\mathrm{T}_{\text {stoch }}=\sigma$ as

$$
\mathrm{T}_{\text {cell }}=\mathrm{T}_{\mathrm{av}}-\sigma \text {. }
$$

On the other hand the red dots in Fig. 2 seem to disobey this interpretation by a Poisson process since the population slopes for PLA cells and stimulated HEK cells are different from one.

We extend our assumptions on the relation between recovery and nucleation to explain these data. We assume that the spike generation rate is 0 only at the time immediately after a spike and relaxes to a constant during the recovery process. A spike might be set off before recovery is complete. Note that this matches well with ideas that the recovery time is set by recovery from channel inhibition, store refilling or similar processes. We study a nucleation rate $\Lambda(t)$ increasing in time $\Lambda(t)=\lambda\left(1-e^{-\rho t}\right)$ where $\rho$ can be interpreted as regeneration rate. Therewith the probability density to observe a $\mathrm{Ca}^{2+}$ transient at time $t$ takes the form

$$
p_{\rho}(t)=\lambda\left(1-e^{-\rho t}\right) \exp \left[-\int_{0}^{t} \lambda\left(1-e^{-\rho t^{\prime}}\right) d t^{\prime}\right],
$$

illustrated in Fig. 3A. With this definition we can calculate the first two moments as

$$
\begin{aligned}
\mathrm{T}_{\mathrm{av}} & =\frac{e^{\frac{\lambda}{\rho}}\left(\frac{\lambda}{\rho}\right)^{1-\frac{\lambda}{\rho}}}{\lambda}\left[\Gamma\left(\frac{\lambda}{\rho}\right)-\Gamma\left(\frac{\lambda}{\rho}, \frac{\lambda}{\rho}\right)\right] \\
\left\langle\mathrm{T}^{2}\right\rangle & =\frac{2 e^{\frac{\lambda}{\rho}}}{\lambda^{2}} \mathcal{F}\left[\left(\frac{\lambda}{\rho}, \frac{\lambda}{\rho}\right),\left(1+\frac{\lambda}{\rho}, 1+\frac{\lambda}{\rho}\right),-\frac{\lambda}{\rho}\right]
\end{aligned}
$$

where $\Gamma(x)$ denotes the Euler $\Gamma$-function, $\Gamma(x, y)$ the incomplete Gamma function and $\mathcal{F}[\mathbf{x}]$ is the generalized hypergeometric function ${ }_{2} \mathrm{~F}_{2}(\mathbf{x})$ [1]. The standard deviation given by

$$
\sigma=\sqrt{\left\langle\mathrm{T}^{2}\right\rangle-\mathrm{T}_{\mathrm{av}}^{2}}
$$


can be calculated and plotted over $\mathrm{T}_{\mathrm{av}}$ in dependence of $\lambda$ and $\rho$ as shown in Fig. 3B, where lines with constant $\lambda$ are obtained by varying $\rho$ and vice versa for lines with constant $\rho$. Note that we did not include a deterministic part $\mathrm{T}_{\text {cell }}$. That would
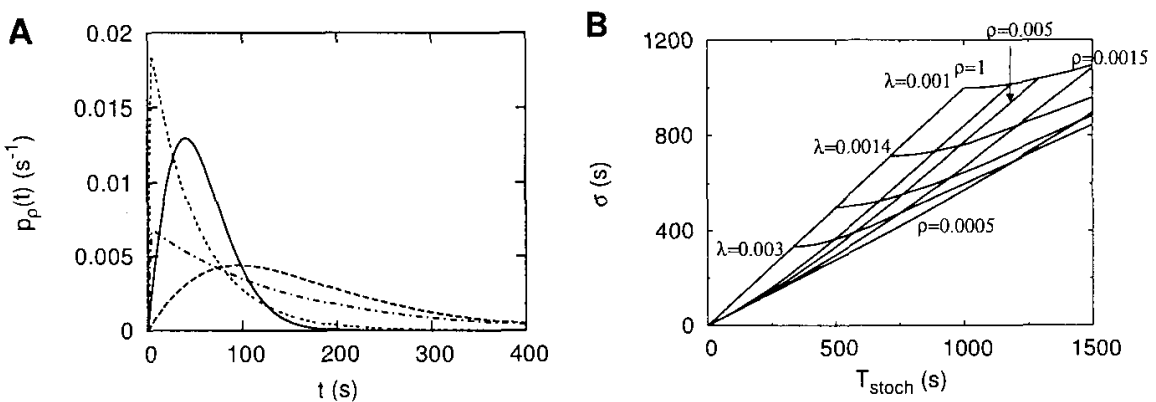

Fig. 3. Influence of a time dependent nucleation rate. A: The density (5) for different parameters. For high values of $\rho p_{\rho}(t)$ merge into a pure Poisson process as shown for $\rho=1$ and $\lambda=0.02$ (dotted line) and $\lambda=0.007$ (dash dotted line). With decreasing $\rho$ the maximum of the distribution is shifted to higher values of $t$. For fixed $\rho=0.01$ the width increases with decreasing $\lambda$ as for the examples $\lambda=0.06$ (solid line) and $\lambda=0.01$ (dashed line). B: The $\sigma-\mathbf{T}_{\text {av }}$ relation in dependence of the nucleation rate $\lambda$ and the regeneration rate $\rho$ given by eqs. (6) and (7). By comparison with the data in Fig. $2 \mathrm{~A}$ we conclude that stimulated HEK cells have high $\lambda$ and small $\rho$.

shift the curves to the right. The analysis shows that for small regeneration rates the slope of the $\sigma-\mathrm{T}_{\mathrm{av}}$ relation decreases and can be approximated for large $\mathrm{T}_{\mathrm{av}}$ and $\sigma$ by the coefficient of variation CV. By inserting (6) and (7) into equation (8) we obtain the functional dependence of the form

$$
m \approx \mathrm{CV}=\frac{\sigma}{\mathrm{T}_{\mathrm{av}}}=f\left(\frac{\lambda}{\rho}\right)
$$

which depends on the ratio $r=\lambda / \rho$ only. From this we conclude that the stimulated HEK cells have high $\lambda$ and small $\rho$. A possible physiological interpretation of this result is the following. The intracellular $\mathrm{IP}_{3}$ concentration is high due to the stimulation so that the $\mathrm{IP}_{3} \mathrm{Rs}$ are sensitized permanently leading to $\mathrm{Ca}^{2+}$ signals arising before cells are in their rest states again.

Nevertheless one might explain the distinct data structure by a large diversity of $\mathrm{T}_{\text {cell }}$. Additionally, we relied on the slope of the linear regression $\left(m_{\text {pop }}\right)$ averaging over the cell populations for information on the slope of the $\sigma$ - $\mathrm{T}_{\mathrm{av}}$ relation. To clarify these issues we performed experiments providing approximations for the slope of the $\sigma-\mathrm{T}_{\mathrm{av}}$ relation. The stochastic part $\mathrm{T}_{\text {stoch }}$ depends on the nucleation rate $\lambda$ and we can consequently characterize the stochastic process by changing $\lambda$ and study the repercussion on $\sigma$ and $\mathrm{T}_{\mathrm{av}} . \lambda$ depends strongly on the concentration of $\mathrm{Ca}^{2+}$ binding proteins (buffers) in the cytosol as they determine the diffusion length of free $\mathrm{Ca}^{2+}$ and hence the strength of spatial coupling between channel clusters [7]. Higher buffer concentrations yield in lower nucleation rates. On the other hand we do not expect 
the small buffer concentrations used to have an influence on the regeneration rate $\rho$ or on the deterministic time $\mathrm{T}_{\text {cell }}$ since both are not very sensitive to the cytosolic buffering capacity. Hence, we predict cells to be shifted on lines corresponding to $\rho=$ constant in Fig. 3B by lowering $\lambda$. We denote the slope of the $\sigma-\mathrm{T}_{\mathrm{av}}$ relation estimated from the data points before and after buffer addition as $m_{\text {shift }}$. If the population slopes before and after adding additional buffer are in the same range as $m_{\text {shift }}$, we can take them as estimate of the slope of the $\sigma-\mathrm{T}_{\mathrm{av}}$ relation.
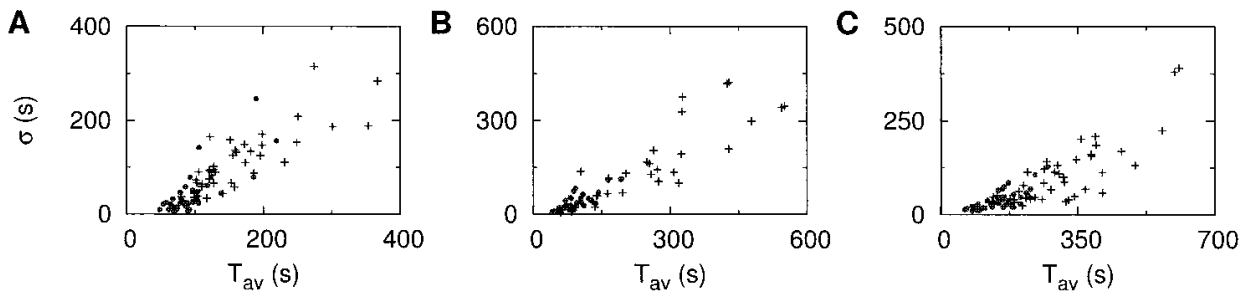

Fig. 4. $\mathrm{Ca}^{2+}$ buffers influence both $\sigma$ and $\mathrm{T}_{\mathrm{av}}$ in astrocytes $(\mathbf{A}, \mathbf{B})$ and in HEK cells $(\mathbf{C})$. For experiments with astrocytes we loaded cells with $20 \mathrm{nM}$ BAPTA (A) as well as with $1 \mu \mathrm{M}$ EGTA (B) showing a similar effect. HEK cells were loaded with $1 \mu \mathrm{M}$ BAPTA (C). For all cell types and buffer concentrations cell seems to be shifted in the direction of the population.

In the experimental realization we measured oscillations in astrocytes and HEK cells for several tens of minutes, loaded additional $\mathrm{Ca}^{2+}$ buffer (BAPTA as well as EGTA) and restarted the measurements. The resulting data are shown in Fig. 4, where again the standard deviation $\sigma$ is plotted over $\mathrm{T}_{\mathrm{av}}$ for astrocytes $(\mathrm{A}, \mathrm{B})$ and HEK cells (C) before (red dots) and after (blue crosses) buffer loading. In the astrocytes experiments we used two distinct buffers, BAPTA (A) and EGTA (B), with different concentrations, namely $20 \mathrm{nM}$ and $1 \mu \mathrm{M}$ respectively, leading to a similar effect, what is in accordance with theoretical predictions [7].

We determined the population slopes $m_{\text {pop }}$ by linear regression and the average shifting slope $m_{\text {shift }}$ for the analysis. We find that the population slopes are quite self consistent and are in good agreement with the shift slopes. Despite the variability of $m_{\text {shift }}$ shown in table 1 , we nevertheless find a separation between the astrocytes and the HEK cells indicating the different dynamical regimes of both populations.

Table 1. Comparison between the population slopes $m_{\text {pop }}^{b}$ before and $m_{\text {pop }}^{a}$ after buffer application and the average shifting slope $m_{\text {shift }}$.

\begin{tabular}{c|ccc}
\hline kind of experiment & $m_{\text {shift }}$ & $m_{\text {pop }}^{b}$ & $m_{\text {pop }}^{a}$ \\
\hline astrocytes with 10 nM BATA & $1.01 \pm 0.16$ & 0.84 & 0.91 \\
astrocytes with $20 \mathrm{nM}$ BATA & $0.94 \pm 0.23$ & 1.04 & 0.89 \\
astrocytes with 1 1 M EGTA & $0.88 \pm 0.19$ & 0.86 & 0.79 \\
HEK cells with 1 $1 \mu \mathrm{M}$ EGTA & $0.49 \pm 0.21$ & 0.56 & 0.58
\end{tabular}


We now return to the decomposition of $\mathrm{T}_{\mathrm{av}}$. From the results above we expect that relation (4) should work for astrocytes, i.e. that the distributions $p\left(\mathrm{~T}_{\text {cell }}\right)$ obtained for data without and with additional buffer should coincide. In addition they should be comparable with the distribution calculated from the intersection of the individual shifting lines with the $\mathrm{T}_{\mathrm{av}}$ axis. The verification is shown in Fig. $5 \mathrm{~A}$, in which the three distribution have a similar shape and posture of their maxima. The naive use of eq. (4) for HEK cells will lead to failure as can be seen in Fig. 5B caused by different parameters for HEK cells in relation (5). Rescaling of $\sigma$ by the inverse of the corresponding $m_{\text {pop }}$ leads again to a nice consistency of the distribution as shown in the inset of $5 \mathrm{~B}$. The small contributions to $p\left(\mathrm{~T}_{\text {cell }}\right)$ with negative $\mathrm{T}_{\text {cell }}$ probably occur due to spike trains which are not yet equilibrated.


Fig. 5. Comparison of distribution densities of $p\left(\mathrm{~T}_{\text {cell }}\right)$ obtained by (4) before and after buffer application (solid and dashed lines respectively) and the density determined by shifting lines (dash dotted lines) for astrocytes (A) loaded with $20 \mu \mathrm{M}$ BAPTA and HEK cells (B) loaded with $1 \mu \mathrm{M}$ BAPTA. The distributions coincide very well for astrocytes, whereas diverge for HEK cells caused by the fact that latter have a time dependent nucleation rate. The inset shows the corrected distributions taking that into account.

We have thus demonstrated that stimulated HEK cells have distinct stochastic properties than spontaneously oscillating astrocytes and that this difference can be estimated by the structure of the population data. Based on that we presented how to extract the intrinsic property $\mathrm{T}_{\text {cell }}$ of a cell out of the global observations $\sigma$ and $\mathrm{T}_{\mathrm{av}}$.

\subsection{Information theory}

In order to interpret the experimental results we are interested in the information distance of a pure (eq. 3) and a time dependent (eq. 5) Poisson process. Therefore we look at the information difference by switching from one process to another one given by the Kullback entropy [8]

$$
\mathcal{K}\left(p_{0}, p_{\mathrm{st}}\right)=k \int_{0}^{\infty} p_{0}(t) \log \frac{p_{0}(t)}{p_{\mathrm{st}}(t)} d t .
$$


By plugging in $p_{0}(t)=p_{\text {poi }}(t)$ and $p_{\text {st }}(t)=p_{\rho}(t)$ into equation (10) we find for the information gain the analytical solution

$$
\mathcal{K}=k\left[H\left(\frac{\lambda}{\rho}\right)+\frac{1}{\left(1+\frac{\lambda}{\rho}\right)}-1\right]
$$

where $H(\lambda / \rho)$ denotes the continuous harmonic series given by

$$
H(x)=\gamma+\frac{d}{d x} \log \Gamma(x+1)
$$

with the Euler-Mascheroni constant $\gamma$ and the Gamma function $\Gamma(x+1)$. That means for $\rho \rightarrow \infty$, i.e. $p_{\rho} \rightarrow p_{\text {poi }}, \mathcal{K}$ goes to zero as expected and for $\rho \ll \lambda$ it turns out that $\mathcal{K} \rightarrow \log (\lambda / \rho)$. Note that in this description the nucleation rates $\lambda$ for both processes were equated. The analysis in the previous section has shown that HEK cells act at higher $\lambda_{\mathrm{s}}$ what additionally increases the information gain and thus expression (11) is a lower estimate.

The expression (11) for $\mathcal{K}$ depends only on the ratio $r=\lambda / \rho$ as the relation for the slope $m$ in eq. (9) does, thus we can estimate the information contained in $\mathrm{Ca}^{2+}$ oscillations by the slope $m$. In Fig. $6 \mathrm{~A}$ the relations (9) and (11) are shown in dependence on $r$ where we used natural units leading to $k=1$. Fig. $6 \mathrm{~B}$ displays the dependence of $\mathcal{K}$ on $m$.
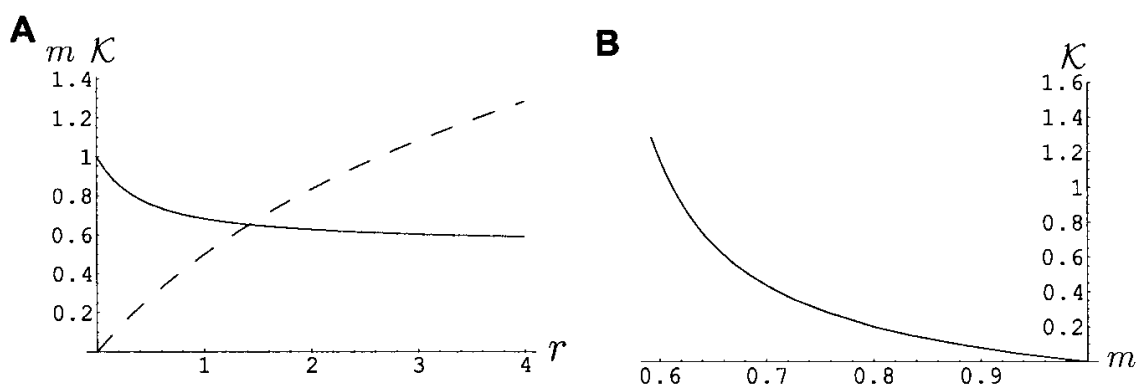

Fig. 6. A: The slope $m \approx \mathrm{CV}$ (solid line) and the information gain $\mathcal{K}$ (dashed line) in dependence of the ratio $r=\lambda / \rho$. B: Due to that relation we can estimate the detectable information in a signal by the experimental population slope $m_{\text {pop }}$ of the $\sigma-T_{a v}$ relation. Values for $\mathcal{K}$ were calculated within natural units, i.e. $k=1$.

The experimental findings make sense in this context. The spontaneous oscillations in astrocytes and microglia might demonstrate the structure of the internal spatial signaling network within cells. The organization of $\mathrm{IP}_{3} \mathrm{R}$ clusters is an optimized design from the information theoretical point of view, since it corresponds to a Poisson process. That is the process with the maximal entropy for processes which are restricted to positive stochastic values with mean $\mu$. Therefore this arrangement is able to detect an eventually occurring signal most efficiently in terms of entropy 
export. Further we can deduce from the data of HEK cells that these cells perform information processing. In Fig. $2 \mathrm{~B}$ we found for the PLA cells a slope $m_{\mathrm{PLA}}=0.7$, which is between the stimulated HEKs and the spontaneous glia cells. That result is suggestive, since these stem cells are in the phase of cell differentiation in which $\mathrm{Ca}^{2+}$ oscillations might control gene expression.

\section{Discussion}

Based on the observation that $\mathrm{Ca}^{2+}$ oscillations occur randomly [13] we here analyzed that stochastic process. The ISI consists of a deterministic part $\mathrm{T}_{\text {cell }}$ and a stochastic part $T_{\text {stoch. }}$. We could extract both of them from global observations. We showed that the stochastic part of spontaneous oscillations obeys a Poisson process, whereas stimulated oscillations can be described by a time dependent nucleation rate. Variability of the deterministic part causes about $25 \%$ of the spread of $\mathrm{T}_{\mathrm{av}}$ of a cell population. The remaining $75 \%$ arise from the stochastic part $\mathrm{T}_{\text {stoch }}$. We have shown by an information theoretical approach that stimulated oscillations indeed carry a signal in comparison to spontaneous behavior.

\section{References}

[1] Abramowitz, M. and Stegun, A., Handbook of Mathematical Functions, 9th edition, Dover Publication, New York, 1970.

[2] Berridge, M., Inositol trisphosphate and calcium signalling, Nature, 361: 315-325, 1993.

[3] Berridge, M., Elementary and global aspects of calcium signalling, J. Physiol., 499, 291-306, 1997.

[4] Berridge, M., Lipp, P., and Bootman, M., The versatility and universality of calcium signalling, Nature Rev. Mol. Cell Biol., 1, 11-22, 2000.

[5] Bootman, M., Niggli, E., Berridge, M., and Lipp, P., Imaging the hierarchical $\mathrm{Ca}^{2+}$ signalling in HeLa cells, J. Physiol, 499, 307-314, 1997.

[6] Falcke, M., On the role of stochastic channel behavior in intracellular $\mathrm{Ca}^{2+}$ dynamics, Biophys. J., 84, 42-56, 2003.

[7] Falcke, M., Reading the patterns in living cells - the Physics of $\mathrm{Ca}^{2+}$ signaling, Advances in Physics, 53, 255-440, 2004.

[8] Goychuk, I. and Hänggi, P., Stochastic resonance in ion channels characterized by information theory, Phys. Rev. E, 61, 4272-4280, 1999.

[9] Marchant, J., Callamaras, N., and Parker, I., Initiation of $\mathrm{IP}_{3}$-mediated $\mathrm{Ca}^{2+}$ waves in Xenopus oocytes, The EMBO J., 18, 5285-5299, 1999.

[10] Marchant, J. and Parker, I., Role of elementary $\mathrm{Ca}^{2+}$ puffs in generating repetitive $\mathrm{Ca}^{2+}$ oscillations, The EMBO Journal, 20, 65-76, 2001.

[11] Putney, J. and Bird, G., The inositolphosphate-calcium signaling system in nonexcitable cells, Endocrine Reviews, 14: 610-631, 1993.

[12] Schuster, S., Marhl, M., and Höfer, T., Modelling of simple and complex calcium oscillations, Eur. J. Biochem., 269, 1333-1355, 2001.

[13] Skupin, A., et al, Constructive use of noise leads to intracellular $\mathrm{Ca}^{2+}$ oscillations, submitted, 2007.

[14] Taylor, C., Inositol trisphosphate receptors: $\mathrm{Ca}^{2+}$-modulated intracellular $\mathrm{Ca}^{2+}$ channels, Biochimica and Biophysica Acta, 1436, 19-33, 1998.

[15] Tsien, R. and Tsien, R., Calcium channels, stores and oscillations, Annu. Rev. Cell Biol., 6, 715-760, 1990. 